\title{
CORRECTIONS
}

\section{Klinefelter's syndrome-a diagnosis mislaid for 46 years}

We have been informed that there is an error in the Clinicians' perspectives section of this Patient's Journey (BMJ 2012;345:e6938, doi:10.1136/bmj.e6938). The first paragraph of this section should have referred to hypergonadotrophic hypogonadism (not "hypogonadotrophic hypogonadism") as a feature of Klinefelter's syndrome.

Cite this as: BMJ 2013;346:f396

๑ BMJ Publishing Group Ltd 2013 